\title{
Dynamic Lane-Changing Trajectory Planning for Autonomous Vehicles Based on Discrete Global Trajectory
}

\author{
Yonggang Liu, Senior Member, IEEE, Bobo Zhou, Xiao Wang, Liang Li, Senior Member, IEEE, Shuo
} Cheng, Zheng Chen, Senior Member, IEEE, Guang Li, Senior Member, IEEE, and Lu Zhang

\begin{abstract}
Automatic lane-changing is a complex and critical task for autonomous vehicle control. Existing researches on autonomous vehicle technology mainly focus on avoiding obstacles; however, few studies have accounted for dynamic lane changing based on some certain assumptions, such as the lane-changing speed is constant or the terminal state is known in advance. In this study, a typical lane-changing scenario is developed with the consideration of preceding and lagging vehicles on the road. Based on the local trajectory generated by the global positioning system, a path planning model and a speed planning model are respectively established through the cubic polynomial interpolation. To guarantee the driving safety, passenger comfort and vehicle efficiency, a comprehensive trajectory optimization function is proposed according to the path planning model and speed planning model. In addition, a dynamic decoupling model is established to solve the problems of real-time application to provide viable solutions. The simulations and real vehicle validations are conducted, and the results highlight that the proposed method can generate a satisfactory lane-changing trajectory for automatic lane-changing actions.
\end{abstract}

Index Terms-Autonomous vehicle, dynamic lane-changing, trajectory planning, real-time optimization, discrete global trajectory

\section{NOMENCLATURE}

\section{A. ACRONYMS}

$\begin{array}{cl}\text { ADAS } & \text { advanced driving assistant systems } \\ \text { PFP } & \text { potential field projection method } \\ \text { RRT } & \text { rapidly exploring random tree } \\ T P & \text { preceding vehicle on the target lane } \\ T L & \text { lagging vehicle on the target lane } \\ P & \text { preceding vehicle on the initial lane } \\ E & \text { the ego vehicle } \\ \text { GPS } & \text { global positioning system } \\ \text { NGSIM } & \text { next generation simulation } \\ \text { SLC } & \text { the static lane-changing } \\ \text { DOLC } & \text { dynamic optimized lane-changing }\end{array}$

This work was supported in part by the National Natural Science Foundation of China (No. 51775063 and 61763021), in part by Chongqing Fundamental Research and Frontier Exploration Project (No. CSTC2018JCYJAX0409), and in part by the EU-funded Marie Skłodowska-Curie Individual Fellowship Project under 845102-HOEMEV-H2020-MSCA-IF-2018. (Corresponding Authors: Zheng Chen and Yonggang Liu)

Y. Liu, B. Zhou and X. Wang are with State Key Laboratory of Mechanical Transmissions \& College of Mechanical and Vehicle Engineering, Chongqing University, Chongqing, 400030, China (e-mail: andylyg@umich.edu, zhoubobo18@163.com, and 20173202013t@cqu.edu.cn).

\author{
DDLC dynamic decoupling lane-changing \\ CPU central processor unit \\ INU inertial navigation unit \\ V2V vehicle to vehicle communication \\ V2I vehicle to infrastructure communication

\section{B. SYMBOLS}

$x_{n}, y_{n}$

the longitude and latitude degree at the $n$th point of initial trajectory

$s_{n} \quad$ the path length at the $n$th point

$s \quad$ the distance along the road

$\rho \quad$ the lateral position perpendicular to $s$ direction

$\rho_{n} \quad$ the planning offset of the nth point

$X_{n}, Y_{n}$ the updated longitude and latitude position at the

${ }_{n}, Y_{n} \quad n$th point of the planed trajectory

$S_{n} \quad$ the path length at $n$th point of the new trajectory

$a, b, c, d$ the coefficients of the cubic polynomial equation

$i, e \quad$ the index of the current and end trajectory point

$s_{i}, \rho_{i} \quad$ the path length and lateral offset at the current

$s_{i}, \rho_{i}$ point

$s_{e}, \rho_{e} \quad$ the path length and lateral offset at the end point

$k_{i}, k_{e} \quad$ the slope of $\rho$ at the current and end point

$n \quad$ the index of arbitrary trajectory points within global

$n$ trajectory points

$k_{n} \quad$ the slope of $\rho$ at the $n$th point

$h_{n}, H_{n}$ the azimuth angle at the nth point of initial and new

${ }_{n}, H_{n}$ trajectory

$e, f, g, h$ the parameters of the velocity cubic polynomial

$v_{i}, v_{e} \quad$ the vehicle speed at the current and end point

$a c c_{i}, a c c_{e}$ the vehicle acceleration at the current and end point

$v_{\text {limit }} \quad$ the road speed limit

$\varsigma \quad$ the adjustable coefficient of the end point speed

$t_{n} \quad$ the time interval between two adjacent points

L. Li and S. Cheng are with State Key Laboratory of Automotive Safety and Energy, Tsinghua University, Beijing, 100084, China (e-mail: liang1@tsinghua.edu.cn, chengs16@mails.tsinghua.edu.cn).

Z. Chen is with Faculty of Transportation Engineering, Kunming University of Science and Technology, Kunming, 650500, China, and School of Engineering and Materials Science, Queen Mary University of London, London, E1 4NS, UK (e-mail: chen@kust.edu.cn)

G. Li is School of Engineering and Materials Science, Queen Mary University of London, London, E1 4NS, UK (e-mail: g.li@qmul.ac.uk)

L. Zhang is with Zhongtong Bus Holding Company, Liaocheng, 252000, China (e-mail: dv900@163.com) 


\begin{tabular}{|c|c|}
\hline$v_{n}$ & the speed of autonomous vehicle at the point $n$ \\
\hline$t_{i}$ & the current time interval \\
\hline$a c c_{n}$ & the acceleration at the trajectory point $n$ \\
\hline$\omega_{\max }$ & the maximum yaw rate \\
\hline$a c c_{(i: e)}$ & the acceleration set from point $i$ to the end point $e$ \\
\hline$a c c_{\max }, b_{E}$ & $\begin{array}{l}\text { the maximum allowable acceleration and } \\
\text { deceleration }\end{array}$ \\
\hline$\kappa_{1}, \kappa_{n}$ & $\begin{array}{l}\text { the curvature at the first and } n \text {th point of the new } \\
\text { trajectory }\end{array}$ \\
\hline$\kappa_{(i: e)}$ & the curvature and speed set from the point $i$ to the \\
\hline$v_{(i: e)}$ & end point $e$ \\
\hline$\omega$ & $\begin{array}{l}\text { the yaw rate set from the current point } i \text { to the end } \\
\text { point } e\end{array}$ \\
\hline$S_{i}, S_{e}$ & $\begin{array}{l}\text { the path length at the current and end point of new } \\
\text { trajectory }\end{array}$ \\
\hline$\eta_{n}$ & $\begin{array}{l}\text { the distance percentage between the trajectory } \\
\text { points } n \text { and } n-1 \text { in the newly planned path }\end{array}$ \\
\hline$\eta$ & the percentage from the point $i$ to the end point $e$ \\
\hline$b_{T P}, b_{T L}$ & $\begin{array}{l}\text { the maximum deceleration of the vehicle } T P \text { and } \\
T L\end{array}$ \\
\hline$v_{T P}, v_{T L}$ & the current speed of the vehicle $T P$ and $T L$ \\
\hline$S A_{T P}$ & $\begin{array}{l}\text { the minimum distance constraint between the } \\
\text { vehicle } E \text { and } T P \text { from the point } i \text { to the end point } e\end{array}$ \\
\hline$S A_{T L}$ & $\begin{array}{l}\text { the minimum distance constraint between the } \\
\text { vehicle } E \text { and } T L \text { from the point } i \text { to the end point } e\end{array}$ \\
\hline$v_{P}$ & the current speed of the vehicle $P$ \\
\hline$D_{P n}$ & the distance from the vehicle $E$ to $P$ at point $n$ \\
\hline$D_{T P n}$ & the distance from the vehicle $E$ to $T P$ at point $n$ \\
\hline$D_{T L n}$ & $\begin{array}{l}\text { the distance from the vehicle } E \text { to } T L \text { at point } n \\
\text { the index of the last trajectory point which belongs }\end{array}$ \\
\hline$m$ & $\begin{array}{l}\text { to the original lane during the lane-changing } \\
\text { process }\end{array}$ \\
\hline$D_{P(i: m)}$ & $\begin{array}{l}\text { the distance between the vehicle } E \text { and } P \text { from point } \\
i \text { to } m\end{array}$ \\
\hline$L_{l w}$ & the lane width \\
\hline$\rho_{m}$ & the lateral offset at the $m$ th point \\
\hline$J_{e}, J_{c}, J_{s}$ & the cost function of efficiency, comfort and safety \\
\hline$\alpha, \beta, \gamma$ & the weighting coefficients of $J_{e}, J_{c}$ and $J_{s}$ \\
\hline$d_{0}$ & a constant distance \\
\hline$t_{h}$ & the time headway \\
\hline$d_{P s}, d_{T P s}$ & the following distance with the vehicle $P$ and $T P$ \\
\hline$t_{l c}$ & the lane-changing duration \\
\hline$k$ & the speed adjustment coefficient \\
\hline$\varphi$ & the adjustment coefficient \\
\hline
\end{tabular}

\section{INTRODUCTION}

Jowadays, high energy conservation efficiency, safety and 1 transportation convenience represent mainstream developing directions in automobile industry [1], and transportation safety is always the top priority when driving vehicles. Statistical studies reveal that $94 \%$ traffic accidents are incurred by driving faults including distraction, fatigue and emotional driving [2]. Autonomous driving technologies provide an effective solution to mitigate these problems and promote automobile development [3]. In particular, advanced driving assistant systems (ADAS), such as adaptive cruise control, autonomous emergency braking and lane keeping assistant, have been widely investigated to reduce potential traffic accidents and improve driving safety [4]. Currently, industry and academia have been actively engaged in development of autonomous vehicles [5], and these enabling technologies can be merged to improve vehicle safety, relieve transportation congestion and optimize driving behavior [6].

The key technologies of autonomous driving mainly include perception, planning and control [7]. Additionally, some investigations are focused on human-automation synergetic driving [8] and interactions between autonomous vehicles and pedestrians [9]. Amongst them, trajectory planning is one of the most important and complex tasks [10], and it can be generally divided into two stages: global planning and local decision [11]. Global planning mainly accounts for trajectory determination of the whole driving route with the help of digital map and local operating system, whilst local trajectory planning can be defined as real-time determination for transition of vehicles from one feasible state to the next state subject to constraints on kinematics capability, comfort, lane boundary, traffic rules and obstacles [12]. Among a series of local decision actions, lanechanging planning is obviously an essential task, and existing planning methods can be grouped into three categories, i.e., artificial potential fields, cell decomposition and optimal control algorithms.

Artificial potential fields mainly consist of attractive fields and repulsive fields in terms of the end goal and obstacles. In Zhou et al.'s research [13], two attractive and repulsive fields corporately operate to achieve path planning of a robot. Hu et al. [14] propose a combined artificial potential field for autonomous driving, and it includes the target, road, lane, vehicle and velocity potentials. The collision avoidance path of autonomous vehicle is determined by the gradient method based on the superposition of different potential functions, and the experiments manifest the feasibility of proposed method on highways. The trajectory of future objects and their associated uncertainties are considered in [15] based on the potential field projection method (PFP), which combines the classical potential field method with a multi-rate Kalman filter estimation. Daily et al. [16] calculate the potential field for a series of circular obstacles inserted into the unobstructed potential field, thereby representing complex shaped obstacles for vehicle path planning. The proposed method can reduce massive computation intensity of the artificial potential field. However, the planned trajectory planned may be trapped in a local minimum, and artificial potential field methods cannot effectively tackle the vehicle kinematic constraints and thus discount the vehicle safety [17].

Cell decomposition divides the surrounding environment into different shaped regions, and each cell represents an obstacle at a corresponding position of environment. In this context, the Dijkstra algorithm is introduced in [18] to determine the set of paths to destinations. In this set, other roads 
in the crossing area are isolated by a virtual border, and the synthetic road is further divided into multiple sections. Zheng et al. [19] add the angle evaluation to the cost function of A-star algorithm, thereby finding the lowest path inflection point to quickly determine the optimal path. In addition, rapidly exploring random tree (RRT) based methods are widely applied in autonomous path planning. In [20], RRT is exploited to conduct the path planning for autonomous vehicles. However, discontinuous characteristics and calculation complexity raised by the method still need to be further investigated [21].

Optimal control algorithms are widely adopted for trajectory planning to find the optimal or sub-optimal trajectory effectively. Werling et al. [22] propose a trajectory generation algorithm based on the optimal control of on-road driving in the presence of dynamic and static obstacles. A polynomial curve is commonly exploited to generate decision trajectories. Zhou et al. [23] adopt the Pontryagin's maximum principle to find the solutions of quadratic optimal control problems and propose a vehicle trajectory planning method for autonomous on-ramp merging operation. In [24], the trajectory is optimized considering safety and smoothness constraints under a rich set of kinematically feasible spatial path candidates generated by the polynomial curve. Hu et al. [11] present a dynamically optimized path planning method based on the cubic spline fitting interpolation and fully takes the static safety, comfortability and dynamic cost into account.

Lane-changing is essentially a complex driving behavior, which may involve actions and reactions of a number of onroad vehicles and show significant impact on driving safety. It is imperative to investigate the lane-changing trajectory planning techniques, which can be divided into two types: static planning and dynamic planning methods. Static planning methods usually plan a trajectory before the lane-changing, and the vehicle will follow the planned trajectory during the whole lane-changing process. Whereas, the dynamic planning method can plan the trajectory with a certain frequency during the lanechanging process. Although the trajectory planning has been widely investigated, most of the researches are mainly focused on avoiding obstacles and yet ignore influences of dynamic surroundings. Cui et al. [25] decompose the lane-changing trajectory to the $\mathrm{x}$-direction and $\mathrm{y}$-direction, and adopt a quintic function to fit the lane-changing trajectory. By this manner, the problem is converted into a constrained optimization problem. Wang et al. [26] propose a trajectory planning method for automated lane-changing operations, and similarly the quintic function is exploited to link the initial position with the final position of the ego vehicle. However, the lane-changing trajectory planning methods mentioned above only consider the instantaneous traffic state and are therefore difficult to cope with the dynamic driving conditions. To the authors' knowledge, only few research findings account for dynamic lane-changing trajectory planning. Luo et al. [27] build an optimized lane-changing trajectory model considering dynamic road constraints. The minimum safe distance is employed to avoid collision during the lane-changing process, thereby promoting adaption to the variation of surrounding vehicles to a certain extent. Even so, the lane-changing trajectory is not dynamically updated. To compensate the drawbacks during the lane-changing process, Yang et al. [28] design a rollover avoidance algorithm, together with a collision avoidance method, to guarantee lane-changing safety; and on this basis, a series of trajectories can be generated during the lane-changing process with a certain frequency to achieve the dynamic trajectory planning. However, the proposed planning model ignores the car-following scenarios before and after lanechanging. Furthermore, the proposed method is difficult to be applied on curvy roads. In conclusion, the discussed methods highlight the following common shortcomings that need to be properly tackled. Firstly, when encountered with curves, their radius should be known a priori, leading to difficulties of online application; secondly, the initial and final lane-changing state is assumed the same, and obviously it cannot be attained all the time in reality; and thirdly, the surrounding vehicles need to be carefully considered to guarantee safety during the lanechanging process. Furthermore, when the state of surrounding vehicles changes suddenly, how to adjust the trajectory timely and plan a new trajectory for returning back to the original lane should also be investigated.

Motivated by these considerations, this study designs a pragmatic dynamic trajectory planning model for lanechanging of autonomous vehicles. First, to cope with the difficulty that the lane-changing segments are not always straight and are hard to be accurately formulated, a flexible lane-changing trajectory planning method is proposed to account for different road geometries. In it, a cubic polynomial model is established based on discrete global trajectory points to generate the lane-changing path. To ensure safety and comfort of the vehicle as well as the efficiency of lane-changing, a comprehensive trajectory optimization function is designed to improve the overall lane-changing performance. To solve the problems in the scope of real-time application, a dynamic decoupling model is established. Finally, simulations and real vehicle validations are conducted, and numerical results highlight that the proposed method can generate a satisfactory lane-changing trajectory. The main contributions of this paper can be attributed to the following three aspects:

(1) A flexible and general lane-changing trajectory planning method is proposed for different road conditions. Even when the geometric information is unknown, the planned trajectory is still proved to be effective.

(2) The proposed lane-changing trajectory planning method achieves the real dynamic lane-changing trajectory planning, and the trajectory is dynamically updated in each iteration. Moreover, when the state of surrounding vehicles changes suddenly and becomes inappropriate for lane-changing, the method can plan a trajectory for returning to the original lane.

(3) The proposed method fully considers car-following behaviors and can solve the speed planning and lane-changing end point, respectively. In addition, the method simplifies the solving process, reduces the calculation complexity and improves the real-time application potential.

The remainder of this paper is structured as follows. Section II introduces the lane-changing trajectory generation module, Section III illustrates the lane-changing trajectory module, Section IV conducts the detailed simulation and real vehicle validation, and finally Section V draws the main conclusions. 


\section{LANE-Changing TRAJectory Generation Module}

A typical lane-changing scenario is shown in Fig. 1, including a preceding vehicle on the target lane, a lagging vehicle on the target lane, a preceding vehicle on the initial lane, and the ego vehicle, which are respectively referred to as $T P$, $T L, P$ and $E$ for simplicity. Note that if the traffic environment is identical for the left lane-changing case and the right lanechanging case, the planning method will generate the same vehicle trajectory except the turning direction. This paper takes the left lane-changing scenario as an example. For ease of tackling the lane-changing trajectory planning problem, two assumptions are imposed here: 1) the vehicle receives the lanechanging command from the upper controller at the current moment [29]; and 2) the lagging vehicle on the target lane will adjust its own speed according to the speed of the ego vehicle. On this basis, the trajectory planning module needs to plan a safe, comfortable and efficient trajectory. To attain it, a multiobjective optimization algorithm for the lane-changing trajectory design is developed, as shown in Fig. 2. First, the lane-changing intention is launched through the upper controller. Second, the candidate trajectory is generated through the coordinate transformation. Then, the candidate trajectory is optimized in real time with the consideration of efficiency, safety and comfort. Finally, the optimal trajectory is implemented to achieve the lane-changing control.

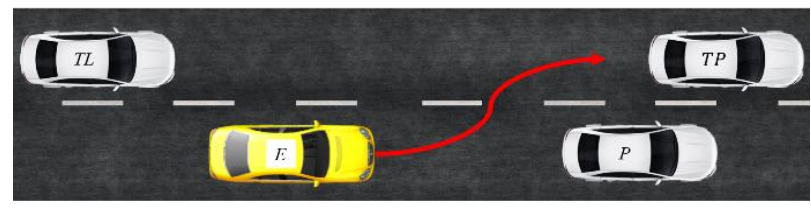

Fig. 1. Autonomous vehicle lane-changing process.

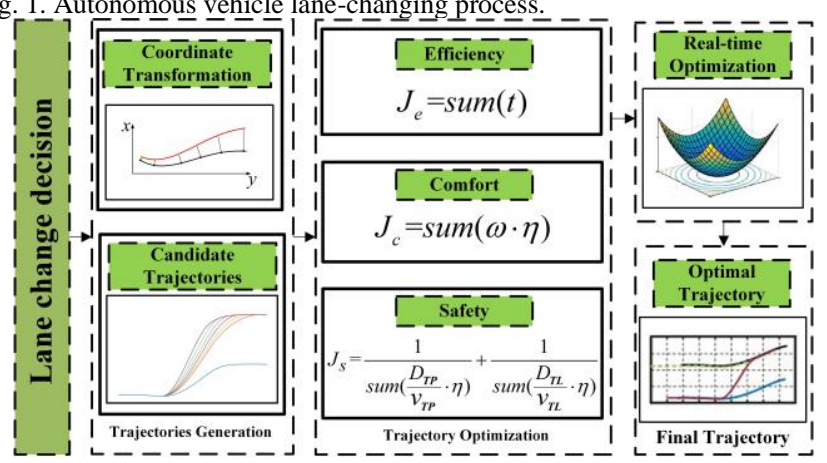

Fig. 2. Flowchart of the proposed path planning method.

\section{A. Coordinate Transformation of Discrete Curve Road}

In this study, a safe and comfortable trajectory from the original lane to the target lane is designed with the compliance of the global route requirement [30]. Actually, the global route is a set of high-precision discrete points of the road center line collected by the global positioning system (GPS). It is a multidimensional array including the information of longitude, latitude and azimuth angle. By numerical conversion, the longitude and latitude are oriented into a high-precision map with the precision of mere multiple centimeters. Generally, the acquired high-precision trajectory consists of random curve points. To facilitate generation of lane-changing path planning under diverse conditions, the path length of each point needs to be solved, as:

$$
\left\{\begin{array}{l}
s_{n}=s_{n-1}+\sqrt{\left(x_{n}-x_{n-1}\right)^{2}+\left(y_{n}-y_{n-1}\right)^{2}} \quad n \geq 2 \\
s_{1}=0
\end{array}\right.
$$

where the subscript $n$ denotes the index of an arbitrary trajectory point within the number of global trajectory points, and the global trajectory points can be collected along the road center line via the GPS in advance. $x_{n}$ and $y_{n}$ denote the longitude and latitude degree at the $n$th point of initial trajectory, respectively; and $s_{n}$ represents the path length at the $n$th point. By solving $s_{n}$, the vehicle position can be mapped from the Cartesian coordinate into the $s-\rho$ coordinate. By this manner, the lane-changing path planning problem can be transformed into the lateral offset of each trajectory in the $s-\rho$ coordinate, as shown in Fig. 3. However, to control the path tracking, the solution should be mapped back to the Cartesian coordinate based on the following transformation, as:

$$
\begin{gathered}
\left\{\begin{array}{l}
X_{n}=x_{n}+\rho_{n} \cdot \sin \left(h_{n}\right) \\
Y_{n}=y_{n}+\rho_{n} \cdot \cos \left(h_{n}\right)
\end{array}\right. \\
\left\{\begin{array}{l}
S_{n}=S_{n-1}+\sqrt{\left(X_{n}-X_{n-1}\right)^{2}+\left(Y_{n}-Y_{n-1}\right)^{2}} \quad n \geq 2 \\
S_{1}=0
\end{array}\right.
\end{gathered}
$$

where $X_{n}$ and $Y_{n}$ denote the updated longitude and latitude position at the $n$th point of the new trajectory, respectively; $\rho_{n}$ denotes the planning offset of the $n$th point; $S_{n}$ represents the path length at $n$th point of new trajectory; $h_{n}$ means the azimuth angle at the $n$th point of initial trajectory and is within $\left(-\frac{3 \pi}{2}, \frac{\pi}{2}\right]$. The related manifestation is shown in Fig. 4 .

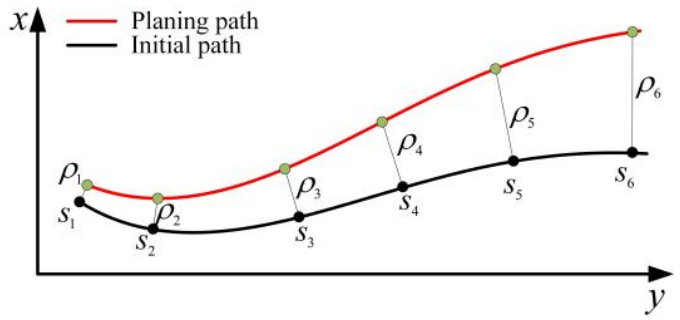

Fig. 3. Coordinate transformation.

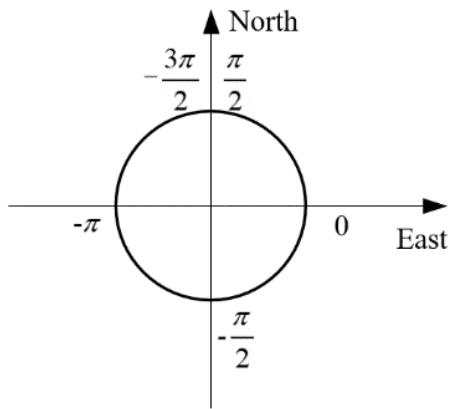

Fig. 4. Coordinate expression of azimuth angle.

\section{B. Path Generation Method}

To generate the lane-changing path, we need to design an algorithm to solve the lateral offset $\rho$. In this study, a cubic 
polynomial function, proposed by Werling et al. [22], is employed to describe the relationship between $\rho$ and $s$ during the lane-changing process. The cubic polynomial function can generate a smooth curve and only entails four parameters. Assuming that the lane width is $L_{l w}, \rho$ can be formulated as:

$$
\rho= \begin{cases}0 & \text { before the lane-change } \\ a \cdot s^{3}+b \cdot s^{2}+c \cdot s+d & \text { in the lane-changing process } \\ L_{l w} & \text { after the lane-change }\end{cases}
$$

And we can also attain:

$$
k= \begin{cases}0 & \text { before the lane-change } \\ 3 a \cdot s^{2}+2 b \cdot s+c & \text { in the lane-changing process } \\ 0 & \text { after the lane-change }\end{cases}
$$

where $k$ denotes the slopes of $\rho$ and $s . k$ and $\rho$ are onedimensional array with the same number of elements as $s . a$, $b, c$ and $d$ denote the coefficients of the cubic polynomial equation. By assuming that the autonomous vehicle shows a qualified tracking capability, the current trajectory point $i$ can be precisely located. Supposing the current lateral offset, the current slope and the current path position are respectively $\rho_{i}$, $k_{i}$ and $s_{i}, a, b, c$ and $d$ can be therefore determined, as:

$$
\left[\begin{array}{l}
\rho_{i} \\
k_{i} \\
\rho_{e} \\
k_{e}
\end{array}\right]=\left[\begin{array}{llll}
s_{i}^{3} & s_{i}{ }^{2} & s_{i} & 1 \\
3 s_{i}{ }^{2} & 2 s_{i} & 1 & 0 \\
s_{e}{ }^{3} & s_{e}{ }^{2} & s_{e} & 1 \\
3 s_{e}{ }^{2} & 2 s_{e} & 1 & 0
\end{array}\right]\left[\begin{array}{l}
a \\
b \\
c \\
d
\end{array}\right]
$$

where $\rho_{e}$, equaling with $L_{l w}$, is the lateral offset at the end point of the lane-changing process. $k_{e}$ means the slope of $\rho$ at the end point and is set to $0 . s_{e}$ denotes the final path position after the lane change. At each time step, $a, b, c$ and $d$ are updated dynamically with $s_{i}$ and $s_{e}$. The solution diagram is shown in Fig. 5. As such, the lane-changing path planning problem can be transformed into the issue of determining the path length of $s_{e}$. The azimuth angle of the planned path $H_{n}$ can be calculated as:

$$
H_{n}=\arctan \left(k_{n}\right)-h_{n}
$$

where $k_{n}$ denotes the slope of $\rho$ and $s$ at the $n$th point.

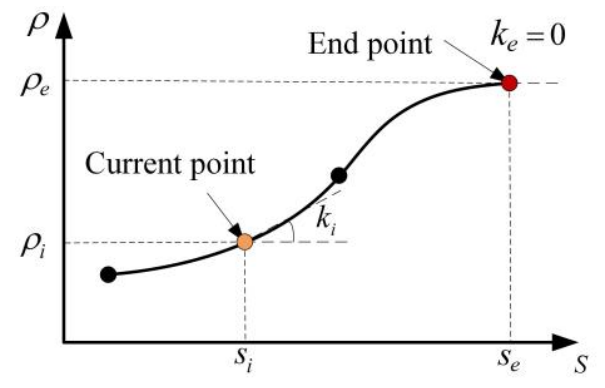

Fig. 5. Solution diagram of cubic polynomial.

\section{Speed Planning Method}

Although some researches have investigated the trajectory planning techniques for dynamic lane-changing, most of emerging works assume that the speed at the end of the lanechanging process is usually supposed to be equal with the initial velocity [27, 28]. However, in real applications, the lanechanging vehicle often adjusts its speed dynamically on the account of safety and comfort concerns during the process. Therefore, it is necessary to establish a dynamic speed planning model in the whole lane-changing process. To ensure that the acceleration is continuous, and the lane-changing process is smooth, similarly, a cubic polynomial model is employed as before. According to the research in [25], the relationship between the vehicle speed and path length during the lanechanging process can be formulated, as:

$$
v=e \cdot s^{3}+f \cdot s^{2}+g \cdot s+h
$$

where $v$ is the planned velocity of the ego vehicle, which is a one-dimensional array and has the same number of elements as $s . e, f, g$ and $h$ are the parameters of the cubic polynomial and can be solved by:

$$
\left[\begin{array}{l}
v_{i} \\
a c c_{i} \\
v_{e} \\
a c c_{e}
\end{array}\right]=\left[\begin{array}{llll}
s_{i}{ }^{3} & s_{i}{ }^{2} & s_{i} & 1 \\
3 s_{i}^{2} & 2 s_{i} & 1 & 0 \\
s_{e}{ }^{2} & s_{e}{ }^{2} & s_{e} & 1 \\
3 s_{e}{ }^{2} & 2 s_{e} & 1 & 0
\end{array}\right]\left[\begin{array}{l}
e \\
f \\
g \\
h
\end{array}\right]
$$

where $v_{i}$ and $a c c_{i}$ denote the current vehicle speed and acceleration that can be acquired from the onboard vehicle control unit. $v_{e}$ and $a c c_{e}$ represent the vehicle speed and acceleration at end point, and $a c c_{e}$ is set to $0 . e, f, g$ and $h$ are dynamically updated with different $s_{i}, s_{e}$ and $v_{e}$. To comply with traffic rules and reduce the calculation amount during optimization, $v_{e}$ needs to be constrained, as:

$$
\left\{\begin{array}{l}
v_{e}<v_{\text {limit }} \\
(1-\varsigma) v_{i}<v_{e}<(1+\varsigma) v_{i} \quad \varsigma \in(0,1)
\end{array}\right.
$$

where $v_{\text {limit }}$ is the road speed limit, and $\varsigma$ is an adjustable coefficient. Obviously, larger $\varsigma$ leads to wider optimization scope. In addition, the destination speed $v_{e}$ needs to meet the acceleration limit and the vehicles speed limit on the target lane, which will be introduced afterwards.

Based on the modeling and analysis discussed previously, the dynamic lane-changing trajectory planning problem is transformed into a problem determining the terminal point $s_{e}$ and terminal speed $v_{\mathrm{e}}$, which will be detailed in the next step.

\section{LANE-ChangIng TRAJECTORY OptimizATION MODULE}

The quality of the planned trajectory is critical and can directly affect the performance of automatic lane changing. In this study, three criteria in terms of efficiency, comfort and safety are adopted to evaluate the performance of the designed lane-changing trajectory in real time. 


\section{A. Efficiency}

As the sketch of the lane-changing process crosses two lanes, long duration of the process not only affects the traffic efficiency, but also increases the potential safety hazard of autonomous vehicles. Thus, it is imperative to endeavor to shorten and optimize the lane-changing duration. The time interval between two adjacent trajectory points in the lanechanging process $t_{n}$ can be expressed as:

$$
\left\{\begin{array}{l}
t_{n}=\frac{\sqrt{\left(X_{n}-X_{n-1}\right)^{2}+\left(Y_{n}-Y_{n-1}\right)^{2}}}{v_{n}} n \in(i, e] \\
t_{i}=0
\end{array}\right.
$$

where $v_{n}$ denotes the speed of the ego vehicle at the point $n$. The efficiency cost function model can be established, as:

$$
J_{e}=\operatorname{sum}(t)
$$

where $J_{e}$ expresses the cost function of efficiency, $t$ indexes the time interval between two adjacent trajectory points from current point $i$ to the end point $e$, and $\operatorname{sum}(t)$ denotes the sum of elements in $t$. Correspondingly, acc at each point can be calculated as:

$$
a c c_{n}=\frac{v_{n}-v_{n-1}}{t_{n}} \quad n \in(i, e]
$$

where $a c c_{n}$ is the acceleration at the trajectory point $n$, and the current acceleration $a c c_{i}$ can be collected through the controller area network communication in real time.

\section{B. Comfort}

To promote comfort during the lane-changing process, the yaw rate and lateral acceleration are considered as constraints, and the cost function is designed considering the yaw rate, as:

$$
\left\{\begin{array}{l}
\max (\omega)<\omega_{\max } \\
\max \left(\operatorname{acc}_{(i: e)}\right)<a c c_{\max } \\
\min \left(\operatorname{acc}_{(i: e)}\right)>-b_{E}
\end{array}\right.
$$

where $a c c_{(i: e)}$ denotes the acceleration set from the current trajectory point $i$ to the ending trajectory point $e$. $\max \left(a c c_{(i: e)}\right)$ and $\min \left(a c c_{(i: e)}\right)$ denote the maximum and minimum value in $a c c_{(i: e)}, a c c_{\max }$ and $b_{E}$ are the maximum allowable acceleration and deceleration of the autonomous vehicle during the lane-changing process. To calculate the yaw rate, the curvature of new planned trajectory needs to be solved based on (10), and thus we can attain:

$$
\left\{\begin{array}{l}
\kappa_{n}=\frac{H_{n}-H_{n-1}}{\sqrt{\left(X_{n}-X_{n-1}\right)^{2}+\left(Y_{n}-Y_{n-1}\right)^{2}}} \quad n \geq 2 \\
\kappa_{1}=0
\end{array}\right.
$$

where $\kappa_{n}$ is the curvature at the $n$th point of the new trajectory. As a result, the yaw rate from the current point to the end position of the lane-changing can be calculated, as:

$$
\omega=\kappa_{(i: e)} \cdot v_{(i: e)}
$$

where $\omega, \kappa_{(i: e)}$ and $v_{(i: e)}$ respectively express the yaw rate, curvature and speed from the current point $i$ to the end point $e$ of the planned new trajectory. The distance percentage between two adjacent trajectory points in the lane-changing process can be yielded, as:

$$
\left\{\begin{array}{l}
\eta_{n}=\frac{\sqrt{\left(X_{n}-X_{n-1}\right)^{2}+\left(Y_{n}-Y_{n-1}\right)^{2}}}{S_{e}-S_{i}} n \in(i, e] \\
\eta_{i}=0
\end{array}\right.
$$

where $\eta_{n}$ denotes the distance percentage between the trajectory points $n$ and $n-1$ in the newly planned path. Now, the comfort cost function $J_{c}$ can be established, as:

$$
\begin{aligned}
& J_{c}=\operatorname{sum}(\omega \cdot \eta) \\
& \text { s.t. }\left\{\begin{array}{l}
\max (\omega)<\omega_{\text {max }} \\
\max \left(a c c_{(i: e)}\right)<a c c_{\text {max }} \\
\min \left(a c c_{(i: e)}\right)>-b_{E}
\end{array}\right.
\end{aligned}
$$

where $\eta$ is the percentage from point $i$ to the end point $e$, and $\operatorname{sum}(\omega \cdot \eta)$ expresses the sum of elements in $\omega \cdot \eta$.

\section{Safety}

In most of the dynamic lane-changing researches, the driving safety is only considered as a constraint, rather than an optimization objective. However, autonomous vehicles should aim to tackle this weakness. Given this, a cost function in terms of safety is considered in this paper. First, a minimum collision avoidance model is designed, as:

$$
\left\{\begin{array}{l}
S A_{T P}=\frac{v_{(i: e)}^{2}}{b_{E}}-\frac{v_{T P}^{2}}{b_{T P}} \\
S A_{T L}=\frac{v_{T L}^{2}}{b_{T L}}-\frac{v_{(i: e)}^{2}}{b_{E}}
\end{array}\right.
$$

where $v_{T P}$ and $v_{T L}$ are the current speed of the vehicle $T P$ and $T L$ shown in Fig. 1, and can be acquired by the perceptual system. $b_{E}, b_{T P}$ and $b_{T L}$ represent the maximum deceleration of the vehicle $E, T P$ and $T L . S A_{T P}$ and $S A_{T L}$ denote the minimum distance constraint between the vehicle $E$ and $T P$, $E$ and $T L$ from the point $i$ to $e$. Here, the speed of vehicle $P, T P$ and $T L$ is assumed to be unchanged for simplicity during the lane-changing process. The distance from the vehicle $E$ to $P, T P$ and $T L$ can be calculated as:

$$
\left\{\begin{array}{l}
D_{P n}=D_{P n-1}+\left(v_{P}-v_{n}\right) t_{n} \\
D_{T P n}=D_{T P_{n-1}}+\left(v_{T P}-v_{n}\right) t_{n} \quad n \in(i, e] \\
D_{T L n}=D_{T L n-1}+\left(v_{n}-v_{T L}\right) t_{n}
\end{array}\right.
$$

where $D_{P n}, D_{T P_{n}}$ and $D_{T L n}$ denote the distance from the vehicle $E$ to $P, T P$ and $T L$ at point $n . D_{P i}, D_{T P i}$, and $D_{T L i}$ are the current distance from the vehicle $E$ to $P, T P$ and $T L$, which can be estimated by the perception module. In addition, the cost function and safety constraints can be formulated, as: 


$$
\begin{array}{ll}
J_{s}= & \frac{1}{\operatorname{sum}\left(\frac{D_{T P}}{v_{T P}} \cdot \eta\right)}+\frac{1}{\operatorname{sum}\left(\frac{D_{T L}}{v_{T L}} \cdot \eta\right)} \\
\text { s.t. }\left\{\begin{array}{l}
\min \left(D_{T P}-S A_{T P}\right)>0 \\
\min \left(D_{T L}-S A_{T L}\right)>0 \\
\min \left(D_{P(i: m)}\right)>0
\end{array}\right.
\end{array}
$$

where $J_{s}$ denotes the cost function of safety. $D_{T P}$ and $D_{T L}$ represent the distance between the vehicle $E$ to $T P$ and $T L$ from the point $i$ to $e . D_{P(i: m)}$ indicates the distance between the vehicle $E$ and $P$ from the point $i$ to $m$, which should be subjected to:

$$
\left\{\begin{array}{l}
\rho_{m+1} \geq \frac{L_{l w}}{2} \\
\rho_{m}<\frac{L_{l w}}{2}
\end{array}\right.
$$

Now, a comprehensive cost function can be established, as:

$$
J=\alpha J_{e}+\beta J_{c}+\gamma J_{s}
$$

where $\alpha, \beta$ and $\gamma$ are the weighting coefficients of $J_{e}, J_{c}$ and $J_{s}$, respectively. $\alpha, \beta$ and $\gamma$ indicate the importance of efficiency, comfort and safety in the integrated optimization algorithm. The larger weighting coefficient indicates that the corresponding criterion is prior to other criteria during the optimization process. For example, if the efficiency factor becomes larger, the trajectory planned by the optimization algorithm will perform lane-changing in a possibly shorter time, and the trajectory length along the $s$ direction will be shorter. Different drivers show different preferences in terms of three factors, e.g., people paying more attention to efficiency expect that the vehicle can finish the lane-changing process in shorter duration. Thus, the coefficient corresponding to efficiency will be enlarged correspondingly. Now, the optimization module can be formulated as:

$$
\begin{array}{ll}
\min & J\left(s_{e}, v_{e}\right) \\
\text { s.t. } & \left\{\begin{array}{l}
\max (\omega)<\omega_{\max } \\
\max \left(a c c_{(i: e)}\right)<a c c_{\max } \\
\min \left(a c c_{(i: e)}\right)>-b_{E} \\
\min \left(D_{T P}-S A_{T P}\right)>0 \\
\min \left(D_{T L}-S A_{T L}\right)>0 \\
\min \left(D_{P(i: m)}\right)>0
\end{array}\right.
\end{array}
$$

Note that the proposed lane-changing trajectory planning method is designated to simultaneously solve the speed planning and path planning. Therefore, both the speed limit and collision-avoidance are correlated with the speed and distance directly. As a result, the optimization method is subject to the constraints on speed and distance, as detailed in (24). By this manner, we can find the optimal lane-changing trajectory with the incorporation of efficiency, comfort and safety model by solving $s_{e}$ and $v_{e}$. By means of the equation derivation detailed previously, it can be concluded that the optimal trajectory is related to $s_{e}$ and $v_{e}$. However, there are three situations that still need to be further tackled:

1) As shown in (24), the lane-changing is a coupling problem, and the optimization function is non-convex and computationally complex, leading to difficulty of real-time operation.

2) Existing optimization models are used only to plan an optimal lane-changing trajectory, whereas they do not consider the vehicle's status under the adaptive cruising condition before and after the lane-changing process.

3) The optimization algorithm may not find a feasible solution; therefore, the lane-changing operation cannot be continued, and the vehicle needs to return back to the original lane. Otherwise, the ego vehicle will be in a dangerous situation. Thus, the proposed lane-changing planning method will not take risks to plan one more trajectory at the future point. Instead, the ego vehicle will continue to perform the lane-changing, until a feasible lane-changing trajectory is generated by the optimization algorithm.

\section{Speed Solution}

In practice, vehicle following actions may exist before and after lane-changing, therefore the speed solution in lanechanging process is based on the traditional adaptive cruise model. First, the following distance needs to be calculated, as:

$$
\left\{\begin{array}{l}
d_{P s}=v_{i} \cdot t_{h} \cdot\left(1-\frac{2 \rho_{i}}{L_{l w}}\right)+d_{0} \\
d_{T P S}=v_{i} \cdot t_{h}+d_{0}
\end{array}\right.
$$

where $d_{P S}$ and $d_{T P S}$ represent the following distance with the vehicle $P$ and $T P$, respectively. $t_{h}$ denotes the time headway, usually set to $2 \mathrm{~s}$, and $d_{0}$ is set to $5 \mathrm{~m}$. The speed of the vehicle $E$ in next step can be determined by:

$v_{i+1}=\left\{\begin{array}{l}\min \left[v_{P}+k \cdot\left(D_{P i}-d_{P_{s}}\right), v_{T P}+k \cdot\left(D_{T P i}-d_{T P s}\right)\right], \rho_{i} \leq \frac{L_{l w}}{2} \\ v_{T P}+k \cdot\left(D_{T P i}-d_{T P s}\right), \rho_{i}>\frac{L_{l w}}{2}\end{array}\right.$

where $k$ is the speed adjustment coefficient and is set to 0.008 .

\section{E. Final Path Position Solution}

The end position of lane-changing $s_{e}$ can be calculated by:

$$
s_{e}=\left\{\begin{array}{l}
v_{i} \cdot\left(1-\frac{\rho_{i}}{L_{l w}+\varphi}\right) \cdot t_{l c}+s_{i}, \text { lane change } \\
v_{i} \cdot \frac{\rho_{i}}{L_{l w}+\varphi} \cdot t_{l c}+s_{i}, \quad \text { back to initial lane }
\end{array}\right.
$$

where $\varphi$ is the adjustment coefficient, $t_{l c}$ is lane-changing time, and is set to $6 \mathrm{~s}$.

To now, we can conclude that a dynamic decoupling algorithm is designed by means of the end point position and speed decoupling technique. In the next step, the simulation and real vehicle test are respectively performed to validate the feasibility of the proposed algorithm. 


\section{VALIDATION AND DISCUSSION}

In this study, the traffic simulation and the real vehicle test are conducted to verify the effectiveness of the proposed trajectory planning method.

\section{A. Simulation Validation}

In this section, the proposed method (referred to as the dynamic decoupling lane-changing (DDLC) method), together with two commonly used lane-changing trajectory planning methods, including the static lane-changing (SLC) method, the dynamic optimized lane-changing (DOLC) method, are employed under the same scenarios for performance comparison. The SLC method is currently mostly applied among all the existing methods, and it assumes that the speed of the preceding and lagging vehicles on the target lane remains unchanged during the lane-changing process. In addition, the SLC method only considers the traffic environment at the decision instant to plan the lane-changing trajectory, which is unable to be adjusted during the whole lane-changing process. Thus, the SLC method can be used only in simple and static scenarios and, therefore, is difficult to plan a feasible and safety trajectory under dynamic and complex conditions $[25,26]$. In contrast, the DOLC method mainly accounts for the variation of environment and updates the lane-changing trajectory dynamically with a certain frequency during the lane-changing process, thereby improving the safety when encountering with complex and dynamic driving environments [27, 28]. However, the DOLC method is time-consuming and does not consider the car-following behavior before and after lane-changing. To overcome the limitations of DOLC method, the proposed DDLC method with the consideration of car-following behavior can solve the speed planning and the lane-changing end point simultaneously, and also the parameter's solving process is simplified. The DDLC method cannot only plan the lanechanging trajectory with a certain frequency under different road conditions, but also enables the vehicle to return back to the original lane under dangerous situations.

To evaluate the feasibility of the proposed algorithm, the traffic simulation is performed in this paper, which includes three typical driving cycles. The driving cycle I is a relatively simple lane-changing scenario, the speed of the preceding vehicle is set to $60 \mathrm{~km} / \mathrm{h}$. The driving cycle II is more complex, the initial speed of the lagging vehicle on the target lane is faster, and the distance to the ego vehicle is shorter. The driving cycle III represents the medium speed and highly dangerous scenario, and the distance among vehicles is quite small. By means of the three simulations, the viability of the planned trajectory can be fully examined.

\section{a. Validation under Driving Cycle I}

In driving cycle I, the lane-changing decision of all methods takes place at $10 \mathrm{~s}$. The speed of vehicle $P$ is set to $60 \mathrm{~km} / \mathrm{h}$, and the speed of $T P$ and $T L$ is randomly selected from the NGSIM dataset, trying to represent the real dynamic traffic environment [31]. The speed variation of the vehicles $P, T P$ and $T L$ can be found in Fig. 7. The comparison results are shown in Figs. 6 to 8. Note that Fig. 6 illustrates the driving path of each vehicle over the entire lane-changing process, rather that the real-time positions. The vehicle trajectories of these three methods are shown in Fig. 6. By the SLC method, the lane-changing process occurs during $10 \mathrm{~s}$ to $16.49 \mathrm{~s}$. The method plans the lanechanging trajectory based only on the state at $10 \mathrm{~s}$, and the speed remains the same during the whole process. Obviously, it is not safe. By the DOLC method, the lane-changing process occurs during $10 \mathrm{~s}$ to $16.77 \mathrm{~s}$. By the DDLC method, the lane-changing process sustains from $10 \mathrm{~s}$ to $17.04 \mathrm{~s}$. The speed of DOLC and DDLC methods changes dynamically according to the traffic environment, as shown in Fig. 7. In this study, the maximum yaw acceleration is introduced to represent the comfort level of the lane-changing process. As shown in Fig. 8, the maximum yaw acceleration values by the SLC, DOLC and DDLC methods under this driving condition are $-1.31 \times 10^{-3} \mathrm{rad} / \mathrm{s}^{2}$, $1.25 \times 10^{-3} \mathrm{rad} / \mathrm{s}^{2}$ and $-1.16 \times 10^{-3} \mathrm{rad} / \mathrm{s}^{2}$, respectively. It indicates that the comfort by the DDLC method is optimal under the driving cycle $\mathrm{I}$. The redundant safety distance is designed as the safety index, as:

$$
\left\{\begin{array}{l}
D S_{P}=D_{P i}-S A_{P} \\
D S_{T P}=D_{T P i}-S A_{T P} \\
D S_{T R}=D_{T R i}-S A_{T R}
\end{array}\right.
$$

The redundant safe distances results are shown in Fig. 9. All the three methods meet the minimum safe distance to complete the lane-changing operation. At about $12 \mathrm{~s}$, the redundant safe distance from the vehicle $T L$ drops sharply, as the vehicle $T L$ accelerates at this time, and the speed is much higher than that of the vehicle $E$. Since the SLC method keeps the speed constant, the minimum redundant safe distance of the SLC method is only $7.60 \mathrm{~m}$. By contrast, the DOLC method calculates the optimal trajectory in real time. There exists obvious acceleration for the vehicle $E$ at $12 \mathrm{~s}$, and the minimum redundant safe distance increases to $12.48 \mathrm{~m}$. By the DDLC method, the vehicle $E$ follows the vehicle ahead to adjust its speed dynamically, and the minimum redundant safety distance is $8.45 \mathrm{~m}$. In terms of safety, the DOLC method outperforms the DDLC method and SLC method under driving cycle I.
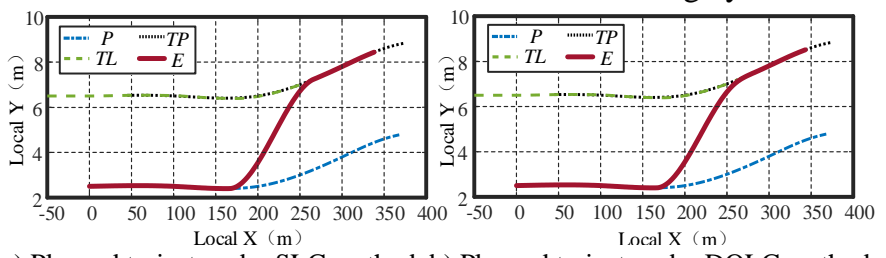

a) Planned trajectory by SLC method b) Planned trajectory by DOLC method
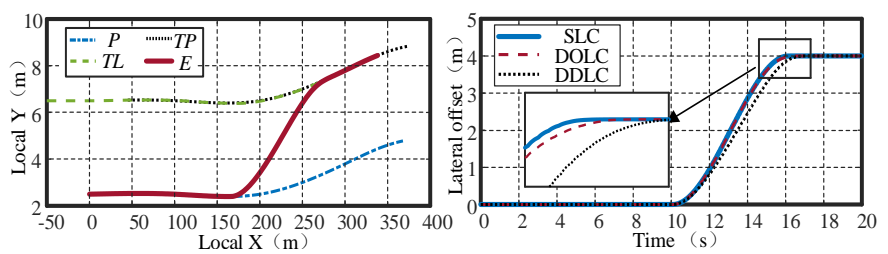

c) Planned trajectory by DDLC method d) Lateral offset of three methods

Fig. 6. Planned trajectory by three methods in driving cycle I.

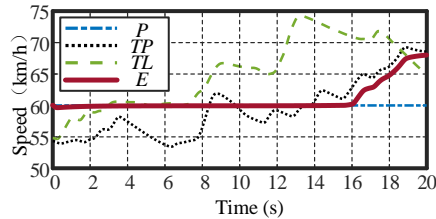

a) Vehicle speed by SLC method

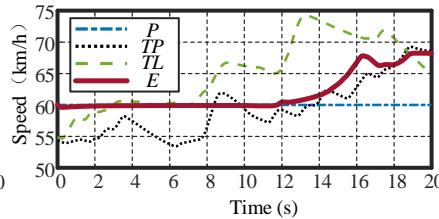

b) Vehicle speed by DOLC method 

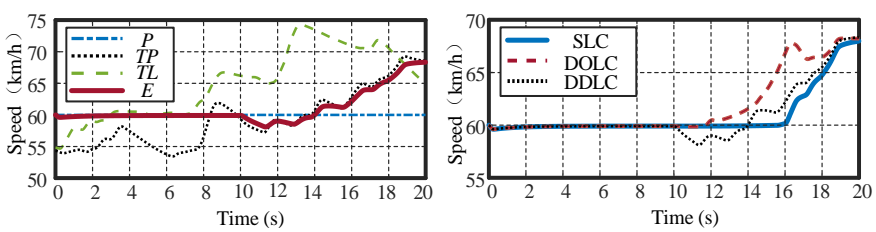

c) Vehicle speed by DDLC method d) Speed of vehicle $E$ by three methods Fig. 7. Vehicle speed by three methods in driving cycle I.

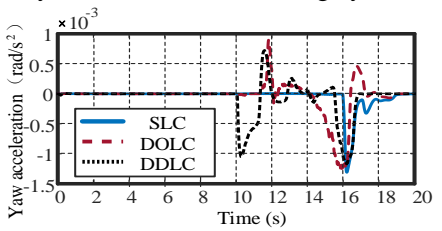

Fig. 8. Yaw acceleration by three methods in driving cycle I.

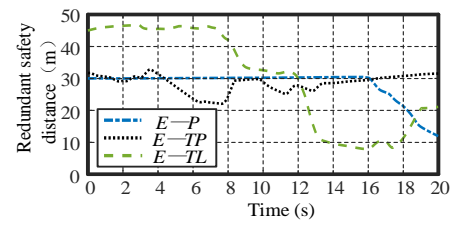

a) Redundant safety distance by SLC method SLC method

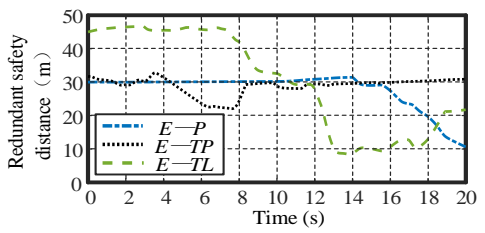

c) Redundant safety distance by DDLC method

Fig. 9. Redundant safety distance by three methods in driving cycle I.

It can be seen from Fig. 7. (c) that the ego vehicle's speed can closely match the putative leading vehicle's speed profile. It indicates that the ego vehicle can adjust its speed according to the environment, thereby meeting the lane-changing safety requirement. In addition, considering the real-time requirement of the proposed algorithm, the calculation time of these three methods in the lane-changing process is statistically analyzed. All the algorithms are conducted on a laptop computer with the central processor unit of Intel Core $17-8750 \mathrm{~h}$ and the memory size of 16 Gigabytes, and the timestep of simulation is $0.01 \mathrm{~s}$. The related statistical results are shown in Table I. Note that the real-time performance denotes the ratio of lane-changing time over the computation time. As can be clearly observed, both the SLC and DDLC methods lead to preferable real-time performance, while the DOLC method shows the worst realtime performance due to the long computation time when conducting online optimization.

TABLE I

COMPUTATIONAL EFFICIENCY UNDER THE DRIVING CYCLE I

\begin{tabular}{cccc}
\hline \hline $\begin{array}{c}\text { Method } \\
\text { category }\end{array}$ & $\begin{array}{c}\text { lane-changing } \\
\text { time }(\mathrm{s})\end{array}$ & $\begin{array}{c}\text { Computing } \\
\text { time }(\mathrm{s})\end{array}$ & Real-time performance \\
\hline SLC & 5.98 & 4.52 & 1.32 \\
DOLC & 6.25 & 70.25 & 0.09 \\
DDLC & 6.85 & 5.93 & 1.16 \\
\hline
\end{tabular}

To comprehensively compare the performance of the methods under the preset driving condition, equation (29) is utilized to calculate the standardized scores of each method, as:

$$
\text { scores }= \begin{cases}\frac{x_{i}}{x_{\max }} \times 100, & \text { positive impact } \\ \frac{x_{\min }}{x_{i}} \times 100, & \text { negative impact }\end{cases}
$$

where $x_{i}$ is calculated under a certain evaluation index. $x_{\max }$ and $x_{\min }$ denote the maximum and minimum values of all methods under the evaluation index, as listed in Table III. It can be concluded that little difference emerges among the three methods in efficiency and comfort under driving cycle I, the SLC method highlights the highest score in lane-changing efficiency, and the DDLC method excels in comfort. The DOLC method shows the optimal performance in safety; however, its real-time performance is extremely poor, making it hardly suitable for practical applications. In short, the proposed DDLC method outperform the other two methods in the overall performance comparison.

TABLE II

THE PERFORMANCE OF EACH METHOD UNDER THE DRIVING CYCLE I

\begin{tabular}{cccc}
\hline \hline \multirow{2}{*}{ Evaluation index } & \multicolumn{3}{c}{ Standardized scores } \\
\cline { 2 - 4 } & SLC & DOLC & DDLC \\
\hline Efficiency & 100.00 & 95.86 & 92.19 \\
Comfort & 88.55 & 92.80 & 100.00 \\
Safety & 60.90 & 100.00 & 67.71 \\
Real-time performance & 100.00 & 6.82 & 87.88 \\
\hline \hline
\end{tabular}

b. Validation under Driving Cycle II

Under driving cycle II, the lane-changing decision of all the methods takes place at $4 \mathrm{~s}$. The initial speed of vehicle $T R$ is faster, and the distance to vehicle $E$ is shorter. All the three methods can plan the smooth lane-changing path, as shown in Fig. 10. As can be found, the lane-changing duration of the SLC method is the longest, $7.82 \mathrm{~s}$, while those of the DOLC and DDLC methods are $6.26 \mathrm{~s}$ and $6.81 \mathrm{~s}$, respectively. It can be seen in Fig. 11 that after the lane-changing decision is made at $4 \mathrm{~s}$, the DOLC and DDLC methods generate a trajectory with an acceleration during the lane-changing process. Whilst, the SLC method always maintains the speed unchanged during the lane-changing process. As shown in Fig. 12, the maximum yaw acceleration value in terms of the SLC, DOLC and DDLC methods under the current driving condition is $-1.20 \times 10^{-3} \mathrm{rad} / \mathrm{s}^{2}$, $-1.59 \times 10^{-3} \mathrm{rad} / \mathrm{s}^{2}$ and $-0.54 \times 10^{-3} \mathrm{rad} / \mathrm{s}^{2}$, respectively. The redundant safe distances results are shown in Fig. 13. The lanechanging process of the SLC method ranges from $4 \mathrm{~s}$ to 11.82 s. At $11.40 \mathrm{~s}$, the lane-changing margin safety distance becomes $-0.28 \mathrm{~m}$, obviously less than zero, thus violating the predefined safety criterion. Hence, the SLC method is unable to plan the route normally under driving cycle II. On the contrary, the DOLC and DDLC methods can acquire the information of the surrounding vehicles in real time and plan the lane-changing trajectory dynamically with a certain frequency. Both methods can gradually increase the surplus safety distance after the lanechanging action begins, and the minimum surplus safety distance is $7.22 \mathrm{~m}$ at the beginning. The calculation time of the three methods under driving cycle II is shown in Table III. It can be seen that the simulation efficiency of each method is relatively close to that under the driving cycle I. The real-time performance of the DOLC method showcases the worst value. 
In addition, the real-time performance of the proposed DDLC method is as good as that of the SLC method.
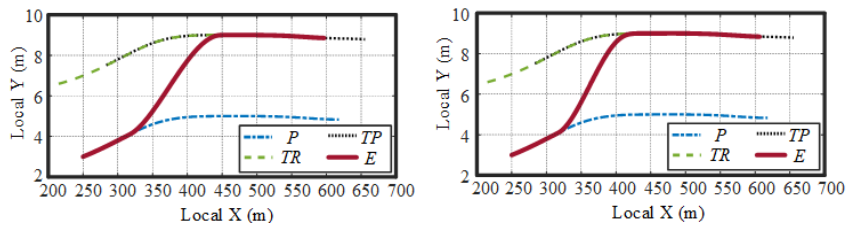

a) Planned trajectory by SLC method b) Planned trajectory by DOLC method
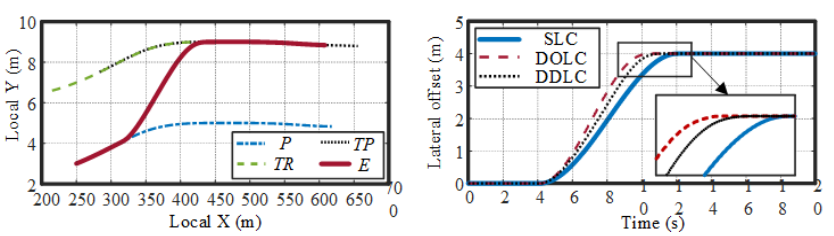

c) Planned trajectory by DDLC method d) Lateral offset of three methods

Fig. 10. Planned trajectory by three methods in driving cycle II.
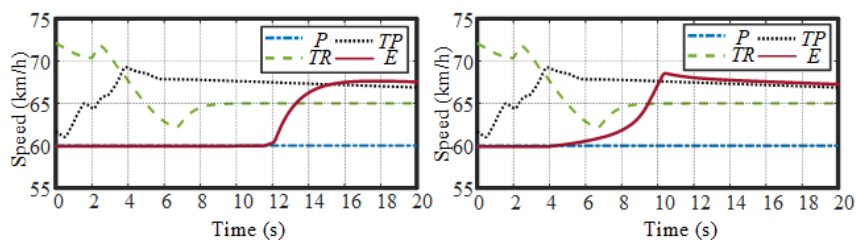

a) Vehicle speed by SLC method

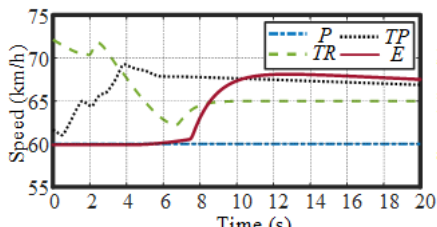

b) Vehicle speed by DOLC method

c) Vehicle speed by DDLC method d) Speed of vehicle $E$ by three methods Fig. 11. Vehicle speed by three methods under driving cycle II.

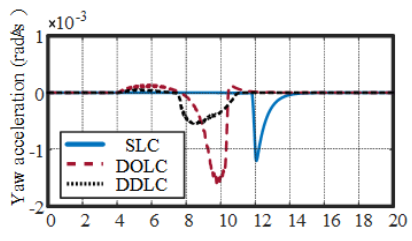

$$
\text { Time (s) }
$$

Fig. 12. The yaw acceleration by three methods under driving cycle II.

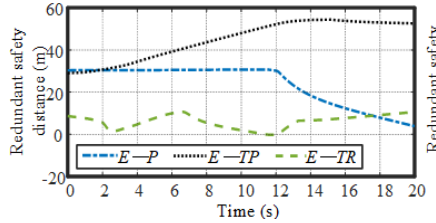

a) Redundant safety distance by SLC method

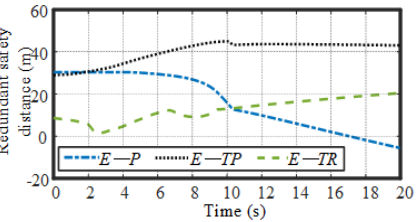

b) Redundant safety distance by DOLC method

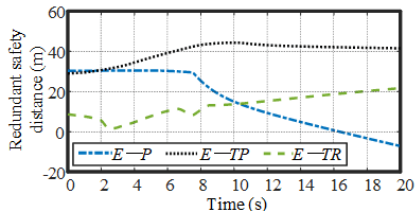

c) Redundant safety distance by DDLC method

Fig. 13. Redundant safe distance by three methods under driving cycle II.

The main evaluation matrices of each method under driving cycle II is listed in Table IV. As can be found, the real-time performance of the SLC method perform best, whereas the safety of lane-changing is unable to meet the conditions. The DOLC method leads to satisfactory performance in efficiency and safety, while the corresponding disadvantages in comfort and real time performance are obvious. The DDLC method raises the optimal performance in terms of safety and comfort, and the lane-changing efficiency and real-time scores are also superior. To sum up, the DDLC method can perform the lanechanging planning with the promising performance and obviously outperform the SLC and DOLC methods.

TABLE III

COMPUTATIONAL EFFICIENCY UNDER DRIVING CYCLE II

\begin{tabular}{ccccc}
\hline \hline $\begin{array}{c}\text { Method } \\
\text { category }\end{array}$ & $\begin{array}{c}\text { lane-changing } \\
\text { time }(\mathrm{s})\end{array}$ & $\begin{array}{c}\text { Computing } \\
\text { time }(\mathrm{s})\end{array}$ & Real-time performance \\
\hline SLC & 7.82 & 7.22 & 1.08 \\
DOLC & 6.26 & 73.06 & 0.09 \\
DDLC & 6.81 & 7.12 & 0.96 \\
\hline \hline \multicolumn{5}{c}{ TABLE IV } \\
THE PERFORMANCE OF EACH METHOD UNDER DRIVING CYCLE II \\
\cline { 2 - 5 } Evaluation index & \multicolumn{3}{c}{ Standardized score } \\
\cline { 2 - 5 } SLC & DOLC & DDLC \\
\hline Efficiency & 80.05 & 100.00 & 91.92 \\
Comfort & 45.00 & 33.96 & 100.00 \\
Safety & 0 & 100.00 & 100.00 \\
Real-time performance & 100.00 & 8.33 & 88.89 \\
\hline \hline
\end{tabular}

\section{c. Validation under Driving Cycle III}

The driving cycle III represents the medium speed and highly dangerous condition. The distance among vehicles is quite small, and the lane-changing decision occurs at $0.5 \mathrm{~s}$. All three methods can design the smooth lane-changing path, as shown in Fig. 14. However, the DDLC method chooses to return back to the original lane, as the surplus safety distance to the following car is less than 0 at $3.38 \mathrm{~s}$. At $9.13 \mathrm{~s}$, the surplus safety distance from the lagging vehicle is greater than 0 . Then, the DDLC method re-executes the lane-changing behavior and completes the lane change at $15.78 \mathrm{~s}$. The lane-changing duration of the SLC, DOLC and DDLC methods is $7.83 \mathrm{~s}, 6.71$ $\mathrm{s}$, and $6.65 \mathrm{~s}$, respectively. The speed change of each method is shown in Fig. 15. As can be found from Fig. 16, the maximum yaw acceleration by the SLC, DOLC, and DDLC methods under this driving condition is $-6.02 \times 10^{-3} \mathrm{rad} / \mathrm{s}^{2},-6.32 \times 10^{-3}$ $\mathrm{rad} / \mathrm{s}^{2}$ and $-6.02 \times 10^{-3} \mathrm{rad} / \mathrm{s}^{2}$, respectively. The three methods show slight difference in comfort index.
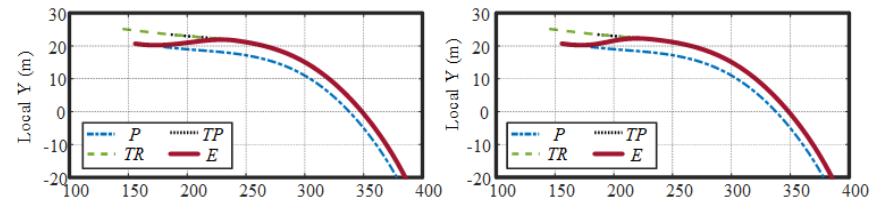

a) Planned trajectory by SLC method b) Planned trajectory by DOLC method
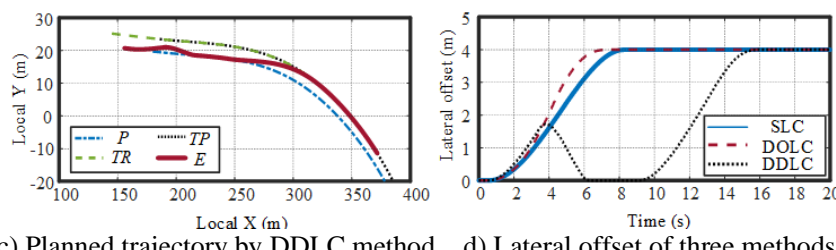

c) Planned trajectory by DDLC method d) Lateral offset of three methods Fig. 14. Planned trajectory by three methods in driving cycle III.

The surplus safety distance under driving cycle III is sketched in Fig. 17. The lane-changing process of the SLC method occurs during $0.5 \mathrm{~s}$ to $8.33 \mathrm{~s}$, and the lane-changing surplus safety distance is less than zero $(-4.85 \mathrm{~m})$ at $3.44 \mathrm{~s}$. Thus, the SLC method cannot meet the requirement of safety. The lane-changing process of the DOLC method occurs during 0.5 $\mathrm{s}$ to $7.21 \mathrm{~s}$. However, at $3.48 \mathrm{~s}$, the lane-changing surplus safety 
distance is less than zero $(-0.70 \mathrm{~m})$. Instead, the DDLC method is the only lane-change trajectory planning method which works under three driving cycles without the violation of safety distance threshold. The time to complete the lane-changing by the DDLC method is from $9.13 \mathrm{~s}$ to $15.78 \mathrm{~s}$, and the minimum surplus safety distance during the lane-changing is $0.02 \mathrm{~m}$ at $9.13 \mathrm{~s}$. By this manner, the superiority and feasibility of the proposed DDLC method is justified.
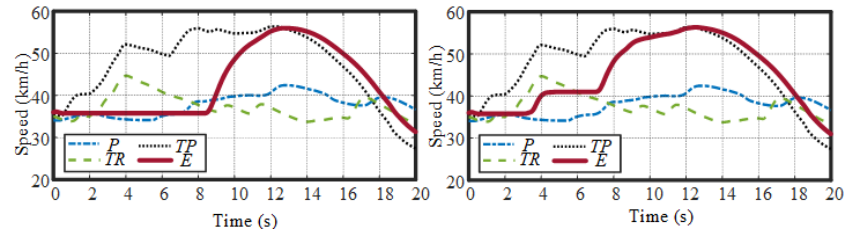

a) Vehicle speed by SLC method
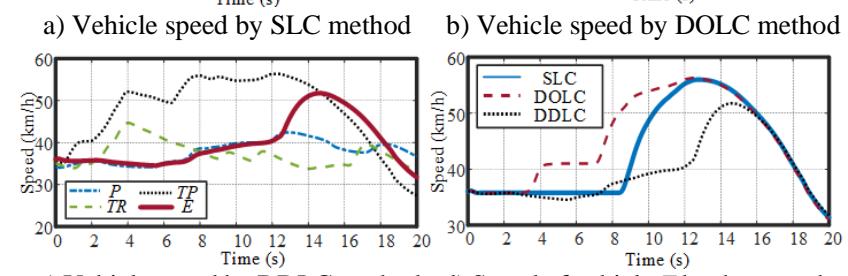

c) Vehicle speed by DDLC method d) Speed of vehicle $E$ by three methods Fig. 15. Vehicle speed by three methods in driving cycle III.

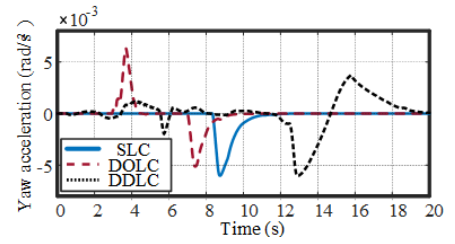

Fig. 16. Yaw acceleration by three methods in driving cycle III.

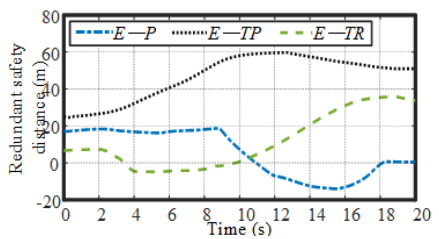

a) Redundant safety distance by SLC method

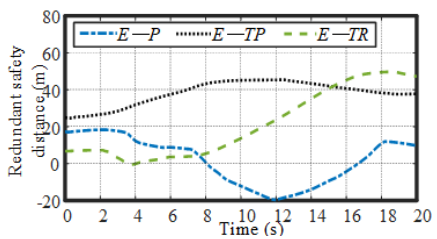

b) Redundant safety distance by DOLC method

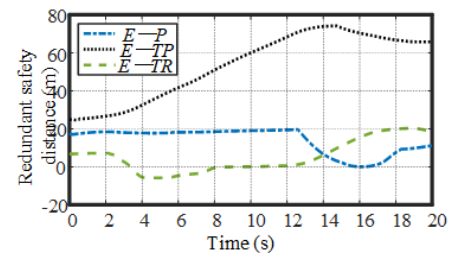

c) Redundant safety distance by DDLC method

Fig. 17. Redundant safety distance by three methods in driving cycle III.

The calculation time of three methods under driving cycle III is listed in Table V. It can be seen that the simulation efficiency of each method is relatively close to that under the driving cycles I and II. The real-time performance of the DOLC method is still the worst. Furthermore, with the increase of the complexity of driving environment, the DDLC method still can achieve preferable real-time performance. The evaluation matrices of each method under the driving cycle III are listed in Table VI, from which we can find that both the SLC and DOLC methods cannot finish the lane-changing safely. Instead, the DDLC method attain the optimal results in terms of safety, comfort and efficiency. Furthermore, the real-time performance is much better than that of the DOLC method.
TABLE V

COMPUTATIONAL EFFICIENCY UNDER DRIVING CYCLE III

\begin{tabular}{ccccc}
\hline \hline $\begin{array}{c}\text { Method } \\
\text { category }\end{array}$ & $\begin{array}{c}\text { lane-changing } \\
\text { time }(\mathrm{s})\end{array}$ & $\begin{array}{c}\text { Computing } \\
\text { time }(\mathrm{s})\end{array}$ & Real-time performance \\
\hline SLC & 7.83 & 3.40 & 2.30 \\
DOLC & 6.71 & 51.52 & 0.13 \\
DDLC & 6.65 & 5.64 & \multicolumn{2}{c}{1.18} \\
\hline \hline \multicolumn{4}{c}{ TABLE VI } \\
THE PERFORMANCE OF EACH METHOD UNDER DRIVING CYCLE III \\
\cline { 2 - 5 } Evaluation index & \multicolumn{3}{c}{ Standardized scores } \\
\cline { 2 - 5 } Efficiency & 84.93 & DOLC & DDLC \\
Comfort & 100.00 & 95.25 & 100.00 \\
Safety & 0 & 0 & 100.00 \\
Real-time performance & 100.00 & 5.65 & 51.30 \\
\hline \hline
\end{tabular}

As safety is the most important criterion during the lanechanging process. The comprehensive score of each method is calculated according to the proportion of 0.2: 0.2: 0.4: 0.2 for efficiency, comfort, safety and real-time performance, as shown in Table VII. Compared with the SLC method, the total score of the DOLC method increases by $1.89 \%$, and the DDLC method increases by $46.41 \%$. Considering the performance under the three driving cycles, the DDLC method shows obvious advances in comprehensive performance, highlighting its full feasibility.

TABLE VII

THE PERFORMANCE OF EACH METHOD UNDER THREE DRIVING CYCLES.

\begin{tabular}{cccc}
\multirow{2}{*}{ Evaluation index } & \multicolumn{3}{c}{ Standardized scores } \\
\cline { 2 - 4 } & SLC & DOLC & DDLC \\
\hline Efficiency & 88.33 & 98.32 & 94.7 \\
Comfort & 77.85 & 74.01 & 100.00 \\
Safety & 20.30 & 66.67 & 89.24 \\
Real-time performance & 100.00 & 6.93 & 76.02 \\
Comprehensive score & 61.36 & 62.52 & 89.54 \\
\hline \hline
\end{tabular}

\section{B. Virtual Scene Co-simulation}

The path tracking with high velocities always remains a challenging task. To verify the traceability of the planned path, a joint simulation is conducted based on MATLAB/Simulink and Carsim. This study employs the Stanley trajectory tracking control strategy detailed in [32], which takes the current position and reference trajectory as the inputs and the target steering wheel angle as the output. The system framework of co-simulation is shown in Fig. 18. The proposed DDLC method updates the lane-changing trajectory based on the information of the surrounding vehicles, ego vehicle and initial trajectory. Then, the lateral controller and PID speed controller respectively calculate the steering and throttle/brake according to the current state and reference trajectory. After that, the virtual scene designed in Carsim shows the final movement.

We record the vehicle motion in the virtual scene, which adopts the same traffic environment as that of the DDLC in simulation. The virtual scenes before, during and after the lanechanging process is shown in Fig. 19. As can be found, the simulation is performed on the curvy road. The tracking performance is shown as Figs. 20 and 21, from which we can find that the planned path can be tracked precisely, indicating that the planned trajectory can meet the requirements of tracking control algorithm. The lateral tracking error is shown in Fig. 21. Compared with the initial trajectory, the maximum lateral error is less than $0.2 \mathrm{~m}$, which is incurred by the larger curvature of the planned lane-changing trajectory. 


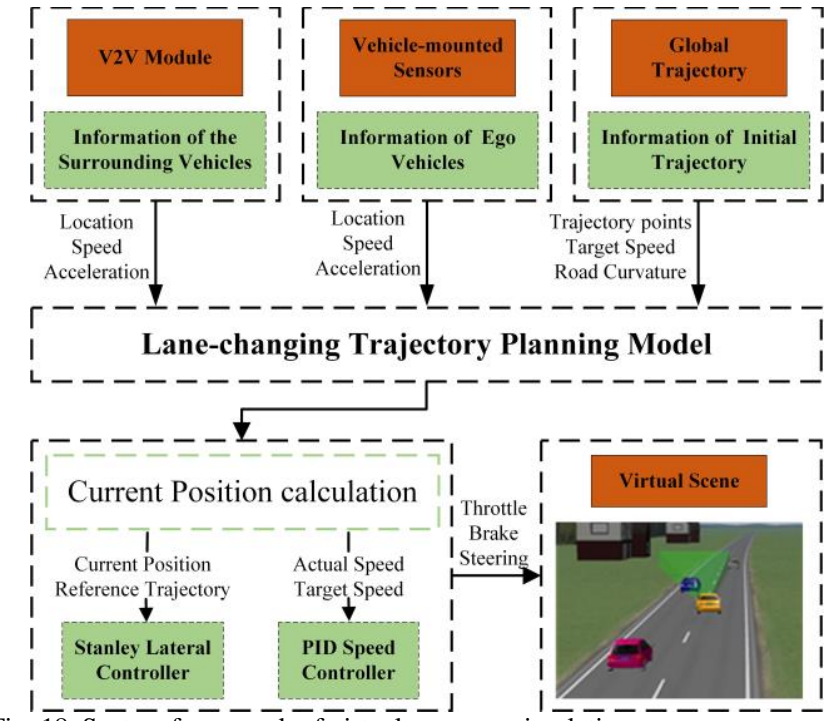

Fig. $\overline{18}$. System framework of virtual scene co-simulation.
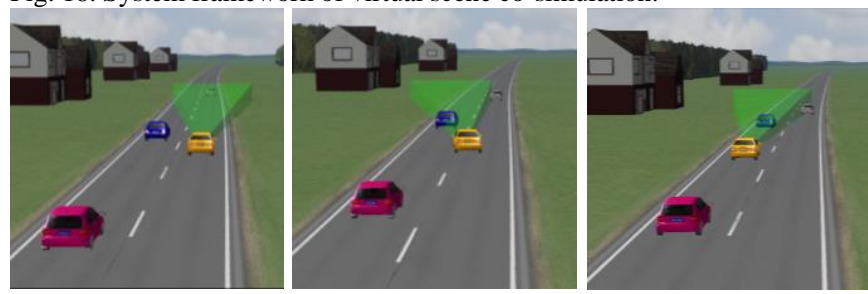

a) Before lane-changing b) During lane-changing c) After lane-changing Fig. 19. The lane-changing scene on the curved road.

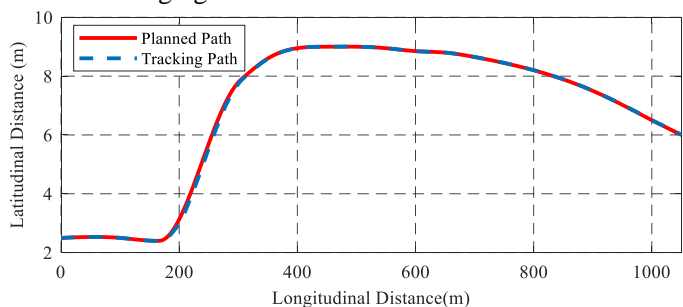

Fig. 20. Comparison of planned path and tracking path.

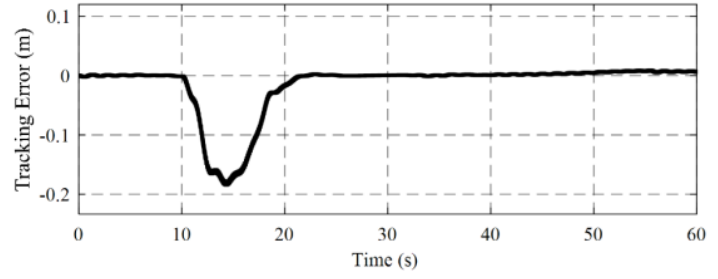

Fig. 21. Lateral tracking error.

\section{Real Vehicle Test and Validation}

We applied the trajectory planning method to a real vehicle. The experimental vehicle is an autonomous bus equipped with GPS, inertial navigation unit (INU), Mobileye and AutoBox of dSPACE, as shown in Fig. 22. Note that since the vehicle to vehicle $(\mathrm{V} 2 \mathrm{~V})$ communication and vehicle to infrastructure (V2I) communication are still under development, we cannot acquire the information of surrounding vehicles. Hence, this study only verifies the feasibility of path planning during the lane-change in real road.

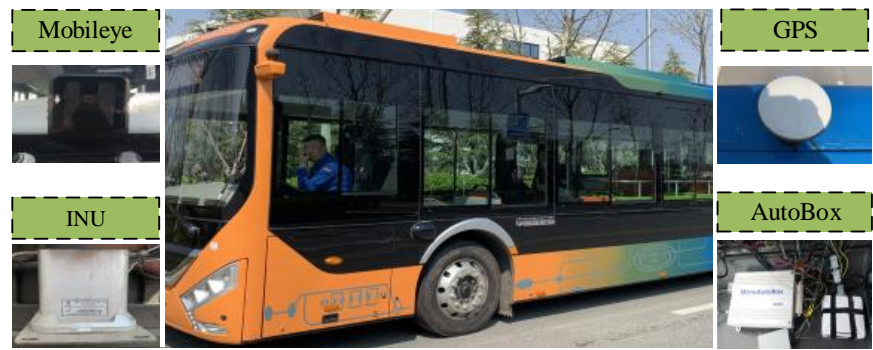

Fig. 22. The test vehicle.

The road experiment was conducted in a prototype development and validation center. As shown in Fig. 23, the global trajectory is an annular road. When there is a stationary vehicle $P$ in front, the ego vehicle makes the decision of lanechanging according to the established rules. An instance of lane-changing process is shown in Fig. 24. As can be seen, the proposed DDLC method can effectively generate a smooth trajectory during the lane-changing process. When the initial speed of vehicle $E$ is $15 \mathrm{~km} / \mathrm{h}$ and the target speed is $30 \mathrm{~km} / \mathrm{h}$, the vehicle $E$ starts to accelerate until it gets close to the vehicle $P$, and then steps into the ACC mode. At $6.3 \mathrm{~s}$, the vehicle $E$ decides to change the lane, the steering angle of $E$ is shown in Fig. 24 (b). Finally, the vehicle completes the lane-changing process safely and successfully. The velocity variation of $E$ is shown in Fig. 24 (c). The whole process was recorded during the experiment, and the channel changing process is shown in Fig. 25. As can be found, the results demonstrate the practicability of the proposed trajectory generation algorithm.

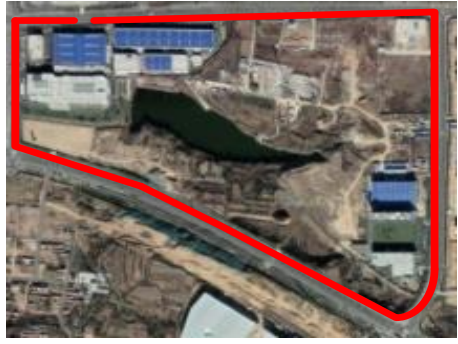

Fig. 23. The experimental road.

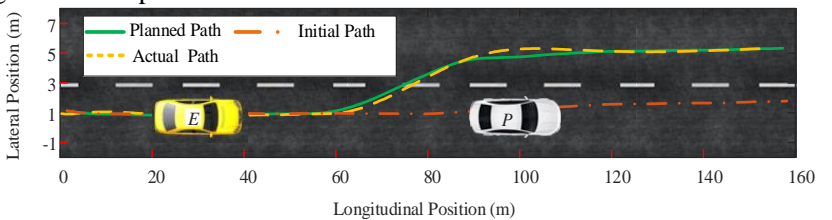

a) The vehicle trajectory

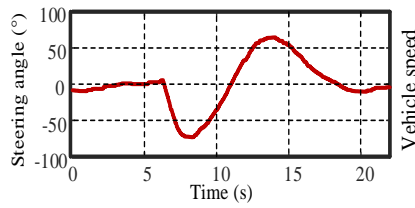

b) Steering angle of lane change Fig. 24. An instance of lane-changing process

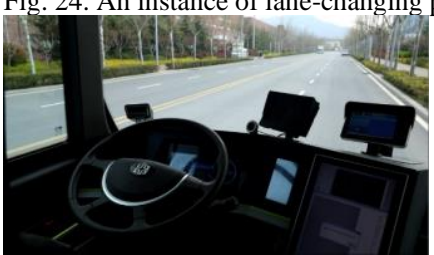

a) Speed up

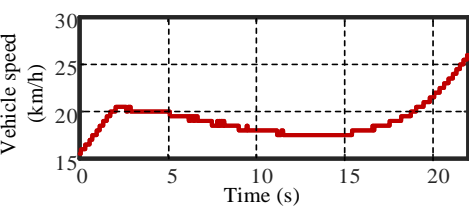

c) Vehicle speed.

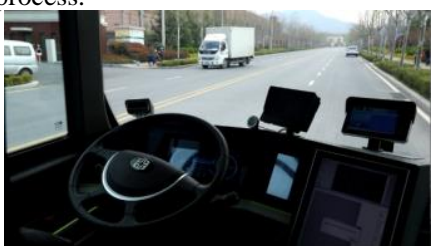

b) Close to the vehicle $P$ 


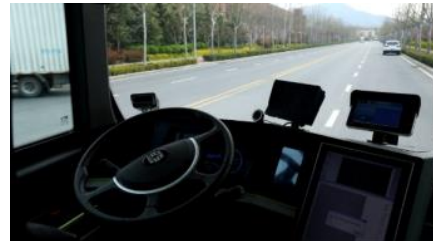

c) Start the lane-changing

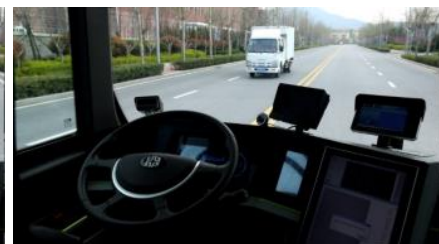

d) Finish the lane-changing

Fig. 25. The whole lane-changing process.

\section{CONCLUSION}

This paper proposes a dynamic lane-changing trajectory planning method based on the discrete global trajectory under complex and dynamic scenarios. The vehicle path and speed of the lane-changing are generated through the cubic polynomial function during the lane-changing process in real time. First, the trajectory generation model is established through the discrete global trajectory information. Then, the dynamically trajectory planning method is proposed, and the corresponding cost function is designed to optimize the generated trajectory in real time with the full consideration of efficiency, safety and comfort. Given the challenges in the dynamic lane-changing trajectory planning, the decoupling lane-changing trajectory planning method is established by means of the vehicle end position and speed decoupling technique. Finally, the joint simulation and real vehicle test are conducted to verify the feasibility and effectiveness of the proposed algorithm. The simulation results demonstrate that the dynamic decoupling lane-changing trajectory planning method exhibits great advances in terms of safety, comfort and efficiency under complex scenarios. The experimental results also highlight the viability of the proposed trajectory generation algorithm. In conclusion, this study solves the real-time lane-changing trajectory optimization based on the actual discrete global trajectory in a complete dynamic environment.

However, there still exist some research limitations and future works required to be addressed. In this research, it is assumed that the sensor can accurately obtain the relative position and motion parameters of the surrounding vehicles. Whereas, some noise will emerge during the actual lanechanging process and will generate a passive impact on the decision-making and trajectory planning. Thus, the sensor noise filtering and multi-source information fusion techniques will be investigated to acquire more precise position in the future research. Moreover, the future trajectory of surrounding vehicles will be predicted before the lane-changing trajectory calculation to improve the robustness and adaptability of the proposed methodology in complex dynamic environment. The fuel consumption of the proposed lane-changing trajectory planning method will also be taken into account in our future research. In addition, it is important to emphasize that we assume the autonomous vehicle can fully track the planned trajectory, and thus a good trajectory tracking controller needs to be developed in the future study.

\section{REFERENCES}

[1] J. Zhang, F.-Y. Wang, K. Wang, W.-H. Lin, X. Xu, and C. Chen, "DataDriven Intelligent Transportation Systems: A Survey," IEEE Transactions on Intelligent Transportation Systems, vol. 12, no. 4, pp. 1624-1639, 2011.

[2] J. Van Brummelen, M. O’Brien, D. Gruyer, and H. Najjaran, "Autonomous vehicle perception: The technology of today and tomorrow,"
Transportation Research Part C: Emerging Technologies, vol. 89, pp. 384406, 2018

[3] W. Zhao, M. Fan, C. Wang, Z. Jin, and Y. Li, "H $\propto$ /extension stability control of automotive active front steering system," Mechanical Systems and Signal Processing, vol. 115, pp. 621-636, 2019.

[4] J. Tang, F. Liu, W. Zhang, R. Ke, and Y. Zou, "Lane-changes prediction based on adaptive fuzzy neural network," Expert Systems with Applications, vol. 91, pp. 452-463, 2018.

[5] B.-J. Chang and J.-M. Chiou, "Cloud Computing-Based Analyses to Predict Vehicle Driving Shockwave for Active Safe Driving in Intelligent Transportation System," IEEE Transactions on Intelligent Transportation Systems, vol. 21, no. 2, pp. 852-866, 2020

[6] D. J. Fagnant and K. Kockelman, "Preparing a nation for autonomous vehicles: opportunities, barriers and policy recommendations," Transportation Research Part A, vol. 77, pp. 167-181, 2015.

[7] C. Wang, Y. Zhang, and W. Zhao, "Multi-objective optimization of a steering system considering steering modality," Advances in Engineering Software, vol. 126, pp. 61-74, 2018.

[8] C. Lv et al., "Characterization of Driver Neuromuscular Dynamics for Human-Automation Collaboration Design of Automated Vehicles," IEEE/ASME Transactions on Mechatronics, vol. 23, no. 6, pp. 2558-2567, 2018.

[9] A. Rasouli and J. K. Tsotsos, "Autonomous Vehicles That Interact With Pedestrians: A Survey of Theory and Practice," IEEE Transactions on Intelligent Transportation Systems, vol. 21, no. 3, pp. 900-918, 2020.

[10]S. Zhang, W. Deng, Q. Zhao, H. Sun, and B. Litkouhi, "Dynamic Trajectory Planning for Vehicle Autonomous Driving," IEEE International Conference on Systems, 2013.

[11]X. Hu, L. Chen, B. Tang, D. Cao, and H. He, "Dynamic path planning for autonomous driving on various roads with avoidance of static and moving obstacles," Mechanical Systems and Signal Processing, vol. 100, pp. 482$500,2018$.

[12]S. Dixit et al., "Trajectory planning and tracking for autonomous overtaking: State-of-the-art and future prospects," Annual Reviews in Control, vol. 45, pp. 76-86, 2018.

[13]Z. Zhou, J. Wang, Z. Zhu, D. Yang, and J. Wu, "Tangent navigated robot path planning strategy using particle swarm optimized artificial potential field," Optik, vol. 158, pp. 639-651, 2018.

[14]H. Hongyu, Z. Chi, S. Yuhuan, Z. Bin, and G. Fei, "An Improved Artificial Potential Field Model Considering Vehicle Velocity for autonomous driving," IFAC-PapersOnLine 2018

[15]M. C. Mora and J. Tornero, "Predictive and Multirate Sensor-Based Planning Under Uncertainty," IEEE Transactions on Intelligent Transportation Systems, vol. 16, no. 3, pp. 1493-1504, 2015.

[16]R. Daily and D. M. Bevly, "Harmonic Potential Field Path Planning for High Speed Vehicles," American Control Conference, 2008.

[17] S. Glaser, B. Vanholme, S. Mammar, D. Gruyer, and L. Nouveliere, "Maneuver-Based Trajectory Planning for Highly Autonomous Vehicles on Real Road With Traffic and Driver Interaction," IEEE Transactions on Intelligent Transportation Systems, vol. 11, no. 3, pp. 589-606, 2010.

[18]R. Kala and K. Warwick, "Multi-Level Planning for Semi-autonomous Vehicles in Traffic Scenarios Based on Separation Maximization," Journal of Intelligent \& Robotic Systems, vol. 72, no. 3-4, pp. 559-590, 2013.

[19] T. Zheng, Y. Xu, and D. Zheng2, "AGV Path Planning based on Improved A-star Algorithm," presented at the 2019 IEEE 3rd Advanced Information Management,Communicates,Electronic and Automation Control Conference, 2019.

[20] J.-H. Ryu, D. Ogay, S. Bulavintsev, H. Kim, and J.-S. Park, "Development of an Autonomous Vehicle for High-speed Navigation and Obstacle Avoidance," Frontiers of Intelligent Autonomous Systems. Springer Berlin Heidelberg, 2013.

[21]D. Gonzalez, J. Perez, V. Milanes, and F. Nashashibi, "A Review of Motion Planning Techniques for Automated Vehicles," IEEE Transactions on Intelligent Transportation Systems, vol. 17, no. 4, pp. 1135-1145, 2016.

[22]M. Werling, J. Ziegler, S. Kammel, and S. Thrun, "Optimal Trajectory Generation for Dynamic Street Scenarios in a Frenet Frame," IEEE International Conference on Robotics and Automation, 2010.

[23]Y. Zhou, M. E. Cholette, A. Bhaskar, and E. Chung, "Optimal Vehicle Trajectory Planning With Control Constraints and Recursive Implementation for Automated On-Ramp Merging," IEEE Transactions on Intelligent Transportation Systems, vol. 20, no. 9, pp. 3409-3420, 2019.

[24] X. Li, Z. Sun, Z. He, Q. Zhu, and D. Liu, "A Practical Trajectory Planning Framework for Autonomous Ground Vehicles Driving in Urban Environments," IEEE Intelligent Vehicles Symposium, 2015. 
[25]Z. Cui, J. Hu and H. Guan, "A lane-changing trajectory planning and assistant decision-making method for autonomous vehicle," 18th COTA International Conference of Transportation Professionals, July 5-8, 2018.

[26] Y. Wang and C. Wei, "A Universal Trajectory Planning Method for Automated Lane-Changing and Overtaking Maneuvers, "Mathematical Problems in Engineering, vol. 2020, pp. 1-13, 2020.

[27] Y. Luo, Y. Xiang, K. Cao, and K. Li, "A dynamic automated lane change maneuver based on vehicle-to-vehicle communication," Transportation Research Part C: Emerging Technologies, vol. 62, pp. 87-102, 2016.

[28]D. Yang, S. Zheng, C. Wen, P. J. Jin, and B. Ran, "A dynamic lanechanging trajectory planning model for automated vehicles," Transportation Research Part C: Emerging Technologies, vol. 95, pp. 228247, 2018.

[29] Y. Liu, X. Wang, L. Li, S. Cheng, and Z. Chen, "A Novel Lane Change Decision-Making Model of Autonomous Vehicle Based on Support Vector Machine," IEEE Access, vol. 7, pp. 26543-26550, 2019.

[30] X. Li, Z. Sun, D. Cao, Z. He, and Q. Zhu, "Real-Time Trajectory Planning for Autonomous Urban Driving: Framework, Algorithms, and Verifications," IEEE/ASME Transactions on Mechatronics, vol. 21, no. 2, pp. 740-753, 2016.

[31](2007). Next Generation Simulation (NGSIM). Available: https://ops.fhwa.dot.gov/trafficanalysistools/ngsim.htm

[32] G. M. Hoffmann, C. J. Tomlin, M. Montemerlo, and S. Thrun, "Autonomous Automobile Trajectory Tracking for Off-Road Driving: Controller Design, Experimental Validation and Racing," presented at the American Control Conference, 2007.

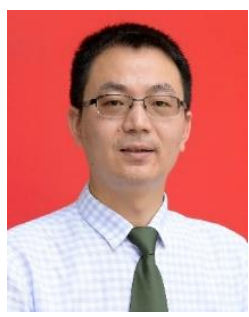

Yonggang Liu, received the B.S and $\mathrm{PhD}$ degrees in Automotive engineering from Chongqing University, Chongqing, China, in 2004 and 2010, while he was a joint PhD of University of Michigan-Dearborn, MI, USA, from 2007 to 2009.

Now he is a Professor with the College of Mechanical and Vehicle Engineering, Chongqing University. His research interests mainly include optimization and control of intelligent Electric Vehicles (EV/HEV) power system, and integrated control of vehicle Automatic Transmissions. He has leaded more than 20 research projects, such as National Natural Science Foundation of China (both Youth Fund and General Program), Ph.D. Programs Foundation of Ministry of Education of China, and China Postdoctoral Science Foundation. More than 70 research papers have been published and 10 patents have been awarded.

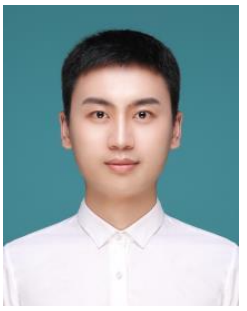

Bobo Zhou received the B.S. degree in Automotive engineering from North University of China, Taiyuan, China, in 2018. He is now working towards the M.S. degree in Automotive engineering at the college of Mechanical and Vehicle Engineering, Chongqing University. Chongqing, China.

His research interests include self-driving car decision-making and trajectory planning.

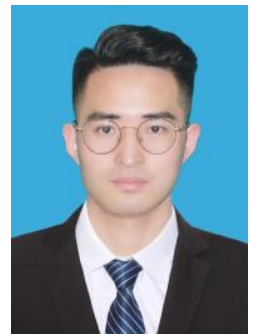

Xiao Wang received the B.S. degree in mechanical engineering from Hefei University of Technology, Hefei China, in 2017, and the M.S. degree in vehicle engineering from Chongqing University. Chongqing China.

His research is focused on intelligent vehicle path planning and control.

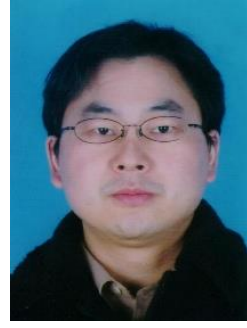

Liang $\mathbf{L i}$ received his Ph.D. degree from the Department of Automotive Engineering at Tsinghua University in 2008.

Since 2017, he has been a tenured professor in Tsinghua University. From November 2011 to December 2012, he was a researcher with the Institute for Automobile Engineering, RWTH Aachen University, Aachen Germany. His research interests mainly include vehicle dynamics \& control, adaptive and nonlinear system control, and hybrid vehicle develop and control. Prof. Li received the China Automotive Industry Science and Technology Progress Award for his achievements in hybrid electrical bus in 2012, and won the National Science Fund for Excellent Young Scholars of the People's Republic of China in 2014.

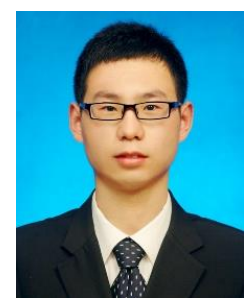

Shuo Cheng received the B.S. degree in mechanical engineering from Harbin Institute of Technology, Harbin, China, in 2016. He is now working towards the $\mathrm{Ph} . \mathrm{D}$. degree in mechanical engineering at the Department of Automotive Engineering, Tsinghua University, Beijing, China.

His research interests include vehicle dynamics states estimation and intelligent vehicle dynamics and control.

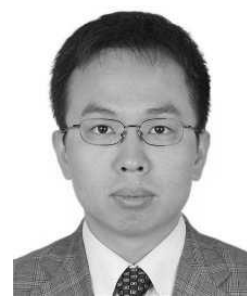

Zheng Chen received the B.S. and M.S. degrees in electrical engineering and the Ph.D. degree in control science engineering from Northwestern Polytechnical University, Xi'an, China, in 2004, 2007, and 2012, respectively. He was a Post-Doctoral Fellow and a Research Scholar with the University of Michigan, Dearborn, MI, USA, from 2008 to 2014.

$\mathrm{He}$ is currently a Professor with the Faculty of Transportation Engineering, Kunming University of Science and Technology, Kunming, Yunnan, China, and also a Marie-Curie Research Fellow with the School of Engineering and Materials Science, Queen Mary University of London, London, UK. He has conducted over 30 projects and has published over 130 peer-reviewed journal papers and conference proceedings. His research interests include battery management and control of intelligent electric vehicles. He was a recipient of the Yunnan Oversea High Talent Project, China, and the second place of IEEE VTS Motor Vehicles Challenge in 2017 and 2018

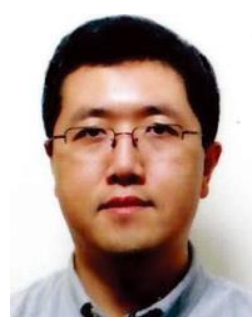

Guang $\mathbf{L i}$ received the $\mathrm{Ph} . \mathrm{D}$. degree in electrical and electronics engineering, specialized in control systems, from the University of Manchester, Manchester, U.K., in 2007.

He is currently a Reader in Dynamics Modeling and Control with the Queen Mary University of London, London, U.K. His research interests include constrained optimal control, model predictive control, adaptive robust control and control applications including renewable energies, energy storage, etc.

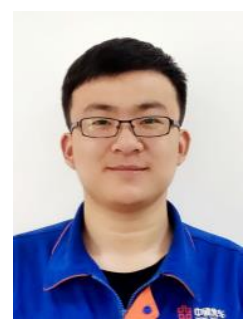

Lu Zhang received his Bachelor Degree in Electrical Engineering and Automation from Qingdao University of Science and Technology, Shandong, China, in 2016. He is currently with Zhongtong Bus Holding Company, Ltd.

$\mathrm{He}$ is mainly responsible for design of electronic control systems and the autopilot control system of electric vehicles. 